\title{
CO-INCIDENCE OF SELECTED EFFECTS OF STROKE - PRELIMINARY FINDINGS
}

\section{WSPÓŁWYSTĘPOWANIE WYBRANYCH NASTĘPSTW UDARU - WYNIKI WSTĘPNE}

\author{
${ }^{1}$ Department of Physiotherapy, Ludwik Rydygier Collegium Medicum in Bydgoszcz, \\ Nicolaus Copernicus University in Toruń, Poland \\ ${ }^{2}$ Neurocognitive Laboratory, Centre for Modern Interdisciplinary Technologies, \\ Nicolaus Copernicus University in Toruń, Poland
}

S u m m a r y

B a ckground. Spasticity, contractures, pusher syndrome, unilateral neglect and heterotopic ossifications are one of the most commonly observed effects in patients after stroke. There is still a need for research on predictors and early identification of such changes in order to minimize their incidence and negative influence to the recovery process.

Objective. To evaluate the incidence and relationship between the selected complications of stroke spasticity, contractures, pusher syndrome, unilateral neglect and heterotopic ossifications.
Results. Among the 141 patients involved in the study, the results were as follows: spasticity was observed in $35.46 \%$, contracture in $42.55 \%$, pusher syndrome in $8.51 \%$, hemispatial neglect in $4.26 \%$ and heterotopic ossifications were observed in $5.67 \%$.

Conclusions. Our findings confirm presented relatively new and important basic and clinical information extending existing studies, especially co-occurrence of selected stroke results and factors influencing their incidence.

\section{Streszczenie}

Tło. Spastyczność, przykurcze, zespół odpychania, zespół zaniedbywania jednostronnego oraz skostnienia heterotopowe są jednymi z najczęściej spotykanych zmian w grupie pacjentów po udarze. Istnieje potrzeba badań nad predykatorami i wczesną identyfikacją ww. zmian, aby zminimalizować ich częstość występowania oraz negatywny wpływ na proces zdrowienia.

$\mathrm{C}$ e 1. Ocenić występowania oraz powiązania pomiędzy wybranymi następstwami udaru: spastycznością, przykurczami, zespołem odpychania, zespołem zaniedbywania jednostronnego oraz skotanieniami heterotopowymi.
Results. Wśród 141 pacjentów włączonych do badania spastyczność została zaobserwowana u 35,46\%, przykurcze u 42,55\%, zespół odpychania u 8,51\%, zespół zaniedbywania jednostronnego u 4,26\%, a skostnienia heterotopowe u 5,67\%.

W n i o ski. Wyniki badania potwierdzają stosunkowo nowe i ważne podstawowe i kliniczne dane rozszerzające wiedzę $\mathrm{z}$ wcześniejszych badań, szczególnie w obszarze współ-występowania badanej grupy następstw udaru oraz czynników wpływających na ich wystąpienie.

Key words: stroke, spasticity, contracture, pusher syndrome, unilateral neglect, heterotopic ossification

Slowa kluczowe: udar, spastyczność, przykurcz, zespół odpychania, zespół zaniedbywania jednostronnego, skostnienie heterotopowe 


\section{INTRODUCTION}

Despite recent advances in knowledge and clinical experience concerning the global stroke epidemic, stroke remains the second leading cause of preventable death and the fourth leading cause of the lost productivity. Risk factors for stroke and their associations are regarded as independent on the country and part of world. Major modifiable risk factors key for prevention of stroke are poor diet, lack of physical activity, obesity, smoking, excessive alcohol intake, lack of hypertension control, diabetes mellitus, atrial fibrillation, dyslipidemia, and stress/depression [1, 2, 3, 4]. Significance of stroke will grow, as the populations' age $[5,6]$. Also, the incidence rate observed in 55 y.o. and younger people was relatively high (13.3\%), and the most common risk factors in this group were smoking, hypertension, dyslipidemia, obesity and diabetes [7].

Despite various advanced prevention approaches and techniques number of post-stroke patients still increases; thus, there is a need to pay particular attention also to stroke causes, results, as far as novel therapies and rehabilitation methods.

Spasticity, contractures, pusher syndrome, unilateral neglect and heterotopic ossifications are one of the most important negative effects of stroke. Their presence may significantly influence the way and effectivity of the therapy; thus, early diagnosis and proper qualification constitute key issue both in inpatient and outpatient treatment, rehabilitation and care. Patients are more severely compromised than those without. Incidence and especially co-incidence of the aforementioned changes often remains uneven or unknown. There is still a need for research on predictors and early identification of such changes in order to minimize their incidence and influence to the recovery process $[8]$.

This study aimed at assessment of the incidence and relationships between selected complications of stroke spasticity, contractures, pusher syndrome, unilateral neglect and heterotopic ossifications.

\section{METHODS}

The objective of the investigation was to evaluate the incidence and relationships between selected complications of stroke spasticity, contractures, pusher syndrome, unilateral neglect and heterotopic ossifications. Thus, study design was observational study.
The research was conducted among 141 adult patients who had undergone stroke. Clinical summary of the patients is presented in table 1.

Table 1. Clinical summary of the patients

\begin{tabular}{|l|c|}
\hline & Patients \\
$\mathrm{n}=141(100 \%)$
\end{tabular}

Each patient was assessed once (at admission, before first session of rehabilitation) using following methods:

- poststroke spasticity (PSS), contracture, and increased muscle tone were measured with the Modified Ashworth Scale and the Tone Assessment Scale $[9,10]$,

- unilateral neglect clinically assessed with the European Stroke Scale [11],

- the Scale for Contraversive Pushing and the Burke Lateropulsion Scale was applied to assess pusher syndrome [12-15],

- heterotopic ossifications were assessed thanks to range-of motion limitations and medical imaging $[16,17]$

Each of the aforementioned methods is assessed as valid, quick, easy-to-use, and commonly used in clinical practice. It allows replication of this study and makes its results useful in everyday clinical practice.

Data were collected using MS Excel 2013. All the data were analyzed using the software Statistica version 12 . Where available the results were calculated as mean, median, minimum value, maximum value and standard deviation. The Shapiro-Wilk test was used as a test for the normality of data. T-student test and Wilcoxon's test were used to compare scores. Spearmen's rho was used to assess correlations. The level of significance was set at $p \leq 0.05$.

The study was conducted in accordance with the Helsinki Declaration and the rules of Good Clinical Practice. Written informed consent was obtained from each patient before the study. 


\section{RESULTS}

Among the 141 patients involved in the study, the results were as follows: spasticity was observed in $35.46 \%$, contracture in $42.55 \%$, pusher syndrome in $8.51 \%$, hemispatial neglect in $4.26 \%$ and heterotopic ossifications were observed in $5.67 \%$ (table 2). Rates of co-incidence were relatively high, especially cooccurrence of spasticity and contractures (29.79\%) (table 2).

Table 2. Co-incidence: percentages in the whole group of patients

\begin{tabular}{|c|c|c|c|c|c|}
\hline & Spasticity & Contracture & \begin{tabular}{|c|} 
Pusher \\
syndrome
\end{tabular} & $\begin{array}{c}\text { Hemispatial } \\
\text { neglect }\end{array}$ & $\begin{array}{l}\text { Heterotopic } \\
\text { ossification }\end{array}$ \\
\hline Sole & 35.46 & 42.55 & 8.51 & 4.26 & 5.67 \\
\hline Spasticity & - & 29.79 & 4.26 & 1.42 & 5.67 \\
\hline Contracture & & - & 7.09 & 3.55 & 4.96 \\
\hline $\begin{array}{c}\text { Pusher } \\
\text { syndrome }\end{array}$ & & & - & 2.84 & 0.71 \\
\hline $\begin{array}{c}\text { Hemispatial } \\
\text { neglect }\end{array}$ & & & & - & 0.71 \\
\hline $\begin{array}{l}\text { Heterotopic } \\
\text { ossification }\end{array}$ & & & & & - \\
\hline
\end{tabular}

Spasticity and contractures were most frequent in women, but pusher syndrome, hemispatial neglect and heterotopic ossifications were most common in men. Rates of co-incidence were relatively high, especially co-occurrence of spasticity and contractures in women $(36.07 \%)$ (table 3$)$

Table 3. Co-incidence: percentages depending on sex

\begin{tabular}{|c|c|c|c|c|c|}
\hline & Spasticity & Contracture & $\begin{array}{c}\text { Pusher } \\
\text { syndrome }\end{array}$ & $\begin{array}{c}\text { Hemispatial } \\
\text { neglect }\end{array}$ & $\begin{array}{l}\text { Heterotopic } \\
\text { ossification }\end{array}$ \\
\hline \multicolumn{6}{|c|}{ Women } \\
\hline Sole & 45.9 & 49.18 & 6.56 & 1.64 & 3.28 \\
\hline Spasticity & - & 36.07 & 3.28 & 1.64 & 3.28 \\
\hline Contracture & & - & 4,92 & 1.64 & 3.28 \\
\hline $\begin{array}{c}\text { Pusher } \\
\text { syndrome }\end{array}$ & & & - & & 0 \\
\hline $\begin{array}{c}\text { Hemispatial } \\
\text { neglect }\end{array}$ & & & & - & 0 \\
\hline $\begin{array}{l}\text { Heterotopic } \\
\text { ossification }\end{array}$ & & & & & - \\
\hline \multicolumn{6}{|c|}{ Men } \\
\hline Sole & 28.75 & 37.5 & 12.5 & 6.25 & 7.5 \\
\hline Spasticity & - & 23.75 & 5 & 1.25 & 7.5 \\
\hline Contracture & & - & 8.75 & 5 & 6.25 \\
\hline $\begin{array}{c}\text { Pusher } \\
\text { syndrome }\end{array}$ & & & - & 5 & 1.25 \\
\hline $\begin{array}{c}\text { Hemispatial } \\
\text { neglect }\end{array}$ & & & & - & 1.25 \\
\hline $\begin{array}{l}\text { Heterotopic } \\
\text { ossification }\end{array}$ & & & & & - \\
\hline
\end{tabular}

Spasticity was equally frequent in all age groups. Contractures, pusher syndrome, hemispatial neglect and heterotopic ossifications were most frequent in younger patients. Rates of co-incidence were relatively high, especially co-occurrence of spasticity and contractures in both age groups (table 4).
Table 4. Co-incidence: percentages depending on age

\begin{tabular}{|c|c|c|c|c|c|}
\hline & Spasticity & Contracture & $\begin{array}{c}\text { Pusher } \\
\text { syndrome }\end{array}$ & $\begin{array}{c}\text { Hemispatial } \\
\text { neglect }\end{array}$ & $\begin{array}{l}\text { Heterotopic } \\
\text { ossification }\end{array}$ \\
\hline \multicolumn{6}{|c|}{ Patients younger than 57 (median) } \\
\hline Sole & 32.35 & 48.53 & 16.18 & 7.35 & 7.35 \\
\hline Spasticity & - & 27.94 & 5.88 & 1.47 & 7.35 \\
\hline Contracture & & - & 11.76 & 5.88 & 5.88 \\
\hline $\begin{array}{c}\text { Pusher } \\
\text { syndrome }\end{array}$ & & & - & 5.88 & 1.47 \\
\hline $\begin{array}{l}\text { Hemispatial } \\
\text { neglect }\end{array}$ & & & & - & 1.47 \\
\hline $\begin{array}{l}\text { Heterotopic } \\
\text { ossification }\end{array}$ & & & & & - \\
\hline \multicolumn{6}{|c|}{ Patients $\geq 57$ y.o. (median) } \\
\hline Sole & 32.88 & 34.25 & 4.11 & 1.37 & 5.48 \\
\hline Spasticity & - & 26.03 & 1.37 & 1.37 & 4.11 \\
\hline Contracture & & - & 2.74 & 1.37 & 4.11 \\
\hline $\begin{array}{c}\text { Pusher } \\
\text { syndrome }\end{array}$ & & & - & 0 & 0 \\
\hline $\begin{array}{l}\text { Hemispatial } \\
\text { neglect }\end{array}$ & & & & - & 0 \\
\hline $\begin{array}{l}\text { Heterotopic } \\
\text { ossification }\end{array}$ & & & & & - \\
\hline
\end{tabular}

Spasticity and pusher syndrome were the most frequent in patients $\leq 4$ weeks after CVA and patients $>12$ months after CVA. Contractures, hemispatial neglect and heterotopic varied, but co-occurrence of spasticity and contractures in patients > 12 months after CVA (table 5).

Table 5. Co-incidence depending on time after CVA

\begin{tabular}{|c|c|c|c|c|c|}
\hline & Spasticity & Contracture & $\begin{array}{c}\text { Pusher } \\
\text { syndrome }\end{array}$ & $\begin{array}{c}\text { Hemispatial } \\
\text { neglect }\end{array}$ & $\begin{array}{l}\text { Heterotopic } \\
\text { ossification }\end{array}$ \\
\hline \multicolumn{6}{|c|}{ Patients $\leq 4$ weeks after CVA } \\
\hline Sole & 36 & 40 & 8 & 0 & 6 \\
\hline Spasticity & - & 30 & 8 & 0 & 4 \\
\hline Contracture & & - & 6 & 0 & 2 \\
\hline $\begin{array}{c}\text { Pusher } \\
\text { syndrome }\end{array}$ & & & - & 0 & 0 \\
\hline $\begin{array}{c}\text { Hemispatial } \\
\text { neglect }\end{array}$ & & & & - & 0 \\
\hline $\begin{array}{l}\text { Heterotopic } \\
\text { ossification }\end{array}$ & & & & & - \\
\hline \multicolumn{6}{|c|}{ Patients > 4 weeks till 3 months after CVA } \\
\hline Sole & 28.21 & 30.77 & 7.69 & 2.56 & 7.69 \\
\hline Spasticity & - & 17.95 & 2.56 & 0 & 5.13 \\
\hline Contracture & & - & 7.69 & 2.56 & 5.13 \\
\hline $\begin{array}{c}\text { Pusher } \\
\text { syndrome }\end{array}$ & & & - & 2.56 & 0 \\
\hline $\begin{array}{c}\text { Hemispatial } \\
\text { neglect }\end{array}$ & & & & - & 0 \\
\hline $\begin{array}{l}\text { Heterotopic } \\
\text { ossification }\end{array}$ & & & & & - \\
\hline \multicolumn{6}{|c|}{ Patients $4-12$ months after CVA } \\
\hline Sole & 29.63 & 59.26 & 14.81 & 11.11 & 7.41 \\
\hline Spasticity & - & 29.63 & 0 & 7.41 & 7.41 \\
\hline Contracture & & - & 14.81 & 11.11 & 7.41 \\
\hline $\begin{array}{c}\text { Pusher } \\
\text { syndrome }\end{array}$ & & & - & 3.7 & 0 \\
\hline $\begin{array}{c}\text { Hemispatial } \\
\text { neglect }\end{array}$ & & & & - & 3.7 \\
\hline $\begin{array}{l}\text { Heterotopic } \\
\text { ossification }\end{array}$ & & & & & - \\
\hline \multicolumn{6}{|c|}{ Patients > 12 months after CVA } \\
\hline Sole & 36 & 48 & 8 & 4 & 8 \\
\hline Spasticity & - & 32 & 0 & 0 & 8 \\
\hline Contracture & & - & 4 & 4 & 8 \\
\hline $\begin{array}{c}\text { Pusher } \\
\text { syndrome }\end{array}$ & & & - & 4 & 0 \\
\hline $\begin{array}{c}\text { Hemispatial } \\
\text { neglect }\end{array}$ & & & & - & 0 \\
\hline $\begin{array}{l}\text { Heterotopic } \\
\text { ossification }\end{array}$ & & & & & - \\
\hline
\end{tabular}


Moderate correlation was observed between spasticity and contractures and between pusher syndrome and hemispatial neglect (table 6). Other correlations, if observed, were rather weak (tables 6-9). Correlations varied depending on sex, age, and time after CVA (tables 6-9).

Table 6. Correlations in the whole group of patients

\begin{tabular}{|c|c|c|c|c|c|}
\hline & Spasticity & Contracture & $\begin{array}{c}\text { Pusher } \\
\text { syndrome }\end{array}$ & $\begin{array}{c}\text { Hemispatial } \\
\text { neglect }\end{array}$ & $\begin{array}{l}\text { Heterotopic } \\
\text { ossification }\end{array}$ \\
\hline Spasticity & - & $\begin{array}{c}0.540 \\
\mathrm{p}=0.000\end{array}$ & n.s. & n.s. & $\begin{array}{c}0.274 \\
\mathrm{p}=0.001\end{array}$ \\
\hline Contracture & & - & $\begin{array}{c}0.204 \\
\mathrm{p}=0.015\end{array}$ & $\begin{array}{c}0.181 \\
\mathrm{p}=0.032\end{array}$ & n.s. \\
\hline $\begin{array}{c}\text { Pusher } \\
\text { syndrome }\end{array}$ & & & - & $\begin{array}{c}0.400 \\
p=0.000\end{array}$ & n.s. \\
\hline $\begin{array}{l}\text { Hemispatial } \\
\text { Neglect }\end{array}$ & & & & - & n.s. \\
\hline $\begin{array}{l}\text { Heterotopic } \\
\text { ossification }\end{array}$ & & & & & - \\
\hline
\end{tabular}

n.s. - not significant

Table 7. Correlations depending on sex

\begin{tabular}{|c|c|c|c|c|c|}
\hline & Spasticity & Contracture & $\begin{array}{c}\text { Pusher } \\
\text { syndrome }\end{array}$ & $\begin{array}{c}\text { Hemispatial } \\
\text { neglect }\end{array}$ & $\begin{array}{l}\text { Heterotopic } \\
\text { ossification }\end{array}$ \\
\hline \multicolumn{6}{|c|}{ Females } \\
\hline Spasticity & - & $\begin{array}{c}0.599 \\
p=0.000\end{array}$ & n.s. & n.s. & n.s. \\
\hline Contracture & & - & n.s. & n.s. & n.s. \\
\hline $\begin{array}{c}\text { Pusher } \\
\text { syndrome }\end{array}$ & & & - & n.s. & n.s. \\
\hline $\begin{array}{c}\text { Hemispatial } \\
\text { neglect }\end{array}$ & & & & - & n.s. \\
\hline $\begin{array}{l}\text { Heterotopic } \\
\text { ossification }\end{array}$ & & & & & - \\
\hline \multicolumn{6}{|c|}{ Males } \\
\hline Spasticity & - & $\begin{array}{c}0.487 \\
\mathrm{p}=0.000\end{array}$ & n.s. & n.s. & $\begin{array}{c}0.355 \\
\mathrm{p}=0.001\end{array}$ \\
\hline Contracture & & - & $\begin{array}{c}0.242 \\
\mathrm{p}=0.030\end{array}$ & n.s. & n.s. \\
\hline $\begin{array}{c}\text { Pusher } \\
\text { syndrome }\end{array}$ & & & - & $\begin{array}{c}0.527 \\
p=0.000\end{array}$ & n.s. \\
\hline $\begin{array}{l}\text { Hemispatial } \\
\text { neglect }\end{array}$ & & & & - & n.s. \\
\hline $\begin{array}{l}\text { Heterotopic } \\
\text { ossification }\end{array}$ & & & & & - \\
\hline
\end{tabular}

Table 8. Correlations depending on age

\begin{tabular}{|c|c|c|c|c|c|}
\hline & Spasticity & Contracture & $\begin{array}{c}\text { Pusher } \\
\text { syndrome }\end{array}$ & $\begin{array}{c}\text { Hemispatial } \\
\text { neglect }\end{array}$ & $\begin{array}{l}\text { Heterotopic } \\
\text { ossification }\end{array}$ \\
\hline \multicolumn{6}{|c|}{ Patients younger than 57 (median) } \\
\hline Spasticity & - & $\begin{array}{c}0.524 \\
p=0.000\end{array}$ & n.s. & n.s. & $\begin{array}{c}0.339 \\
p=0.004\end{array}$ \\
\hline Contracture & & - & n.s. & n.s. & n.s. \\
\hline $\begin{array}{c}\text { Pusher } \\
\text { syndrome }\end{array}$ & & & - & $\begin{array}{c}0.488 \\
p=0.000\end{array}$ & n.s. \\
\hline $\begin{array}{c}\text { Hemispatial } \\
\text { neglect }\end{array}$ & & & & - & n.s. \\
\hline $\begin{array}{l}\text { Heterotopic } \\
\text { ossification }\end{array}$ & & & & & - \\
\hline \multicolumn{6}{|c|}{ Patients $\geq 57$ y.o. (median) } \\
\hline Spasticity & - & $\begin{array}{c}0.629 \\
p=0.000\end{array}$ & n.s. & n.s. & n.s. \\
\hline Contracture & & - & n.s. & n.s. & n.s. \\
\hline $\begin{array}{c}\text { Pusher } \\
\text { syndrome }\end{array}$ & & & - & n.s. & n.s. \\
\hline $\begin{array}{c}\text { Hemispatial } \\
\text { neglect }\end{array}$ & & & & - & n.s. \\
\hline $\begin{array}{l}\text { Heterotopic } \\
\text { ossification }\end{array}$ & & & & & - \\
\hline
\end{tabular}

Table 9. Correlations depending on time after CVA

\begin{tabular}{|c|c|c|c|c|c|}
\hline & Spasticity & Contracture & $\begin{array}{c}\text { Pusher } \\
\text { syndrome }\end{array}$ & $\begin{array}{c}\text { Hemispatial } \\
\text { neglect }\end{array}$ & $\begin{array}{l}\text { Heterotopic } \\
\text { ossification }\end{array}$ \\
\hline \multicolumn{6}{|c|}{ Patients $\leq 4$ weeks after CVA } \\
\hline Spasticity & - & $\begin{array}{c}0.663 \\
p=0.000\end{array}$ & $\begin{array}{c}0.393 \\
\mathrm{p}=0.004\end{array}$ & n.s. & n.s. \\
\hline Contracture & & - & n.s. & n.s. & n.s. \\
\hline $\begin{array}{c}\text { Pusher } \\
\text { syndrome }\end{array}$ & & & - & n.s. & n.s. \\
\hline $\begin{array}{c}\text { Hemispatial } \\
\text { neglect }\end{array}$ & & & & - & n.s. \\
\hline $\begin{array}{l}\text { Heterotopic } \\
\text { ossification }\end{array}$ & & & & & - \\
\hline \multicolumn{6}{|c|}{ Patients > 4 weeks till 3 months after CVA } \\
\hline Spasticity & - & $\begin{array}{c}0.446 \\
p=0.004\end{array}$ & n.s. & n.s. & n.s. \\
\hline Contracture & & - & n.s. & n.s. & n.s. \\
\hline $\begin{array}{c}\text { Pusher } \\
\text { syndrome }\end{array}$ & & & - & $\begin{array}{c}0.562 \\
\mathrm{p}=0.000\end{array}$ & n.s. \\
\hline $\begin{array}{c}\text { Hemispatial } \\
\text { neglect }\end{array}$ & & & & - & n.s. \\
\hline $\begin{array}{l}\text { Heterotopic } \\
\text { ossification }\end{array}$ & & & & & - \\
\hline \multicolumn{6}{|c|}{ Patients $4-12$ months after CVA } \\
\hline Spasticity & - & $\begin{array}{c}0.538 \\
\mathrm{p}=0.004\end{array}$ & n.s. & n.s. & $\begin{array}{c}0.436 \\
\mathrm{p}=0.023\end{array}$ \\
\hline Contracture & & - & n.s. & n.s. & n.s. \\
\hline $\begin{array}{c}\text { Pusher } \\
\text { syndrome }\end{array}$ & & & - & n.s. & n.s. \\
\hline $\begin{array}{c}\text { Hemispatial } \\
\text { neglect }\end{array}$ & & & & - & n.s. \\
\hline $\begin{array}{l}\text { Heterotopic } \\
\text { ossification }\end{array}$ & & & & & - \\
\hline \multicolumn{6}{|c|}{ Patients > 12 months after CVA } \\
\hline Spasticity & - & $\begin{array}{c}0.614 \\
p=0.001\end{array}$ & n.s. & n.s. & n.s. \\
\hline Contracture & & - & n.s. & n.s. & n.s. \\
\hline $\begin{array}{c}\text { Pusher } \\
\text { syndrome }\end{array}$ & & & - & $\begin{array}{c}0.692 \\
p=0.000\end{array}$ & n.s. \\
\hline $\begin{array}{c}\text { Hemispatial } \\
\text { neglect }\end{array}$ & & & & - & n.s. \\
\hline $\begin{array}{l}\text { Heterotopic } \\
\text { ossification }\end{array}$ & & & & & - \\
\hline
\end{tabular}

\section{DISCUSSION}

Author aimed at presentation of the incidence and relationships between the selected negative effects of stroke: spasticity, contractures, pusher syndrome, unilateral neglect and heterotopic ossifications. Such comprehensive approach is rare.

Low number of relevant studies makes compartmental studies difficult. Results of this study are similar to those published by other authors, but more detailed. Spasticity was observed in $35.46 \%$, contractures in $42.55 \%$, pusher syndrome in $8.51 \%$, hemispatial neglect in $4.26 \%$ and heterotopic ossifications in $5.67 \%$ of patients. Incidence varies depending on sex, age, and time after CVA. According to the literature, prevalence of PSS ranges from $4 \%$ to $42.6 \%$, including $4-27 \%$ in patients $1-4$ weeks poststroke, $19-26.7 \%$ in patients 1-3 months poststroke, and $17-42.6 \%$ in patients $>3$ months poststroke [18]. Incidence of the unilateral neglect in patients with acute stroke was assess by SiekierkaKleiser et al.: $32.69 \%$ [19]. Pusher syndrome was 
observed in 9.4-28\% post-stroke survivors [20-22]. According to the recent review of Allison et al., incidence of spasticity in poststroke survivors with weakness ranged from $33 \%$ to $78 \%$, and contractures were present in $>50 \%$. Related impairments and their co-occurrence is relatively common and may negatively influence the time course of the therapy. There is a need to admit that spasticity may appear within 1 week after the stroke, and contracture within two weeks, and continue to develop over 3-6 months [23]. Thus, clinicians should be prepared not only to intervene early but also act over a prolonged time period of recovery.

The new and important aspects of the study are rates of co-incidence were relatively high, especially co-occurrence of spasticity and contractures. Reported co-occurrence varies depending on sex, age, and time after CVA. This report is reported as preliminary and very general - values of measurements and their analysis will be reported in future detailed publications.

Limitations of current study may constitute used diagnostic methods and low number of factors regarded as predictors of selected complications of stroke which may limit the strength of the findings. Many prognostic signs should be taken into consideration. To optimize the final evaluation there is need to use the combinations of both factors and complications. There is an intention to continue this study on bigger sample of patients based on randomized controlled trail design. Further studies are needed to examine increased difficulty of the therapy, rehabilitation and care associated with co-occurrence of the described post-stroke impairments to enable multidisciplinary therapeutic team to develop targeted interventions and achieve therapeutic goal(s).

\section{CONCLUSIONS}

Our findings confirm presented new and important basics and clinical information extending the existing studies, especially co-occurrence of complications and factors influencing their incidence.

\section{ACKNOWLEDGEMENTS}

InterDoCTor project and Neurocognitive Laboratory in Centre for Modern Interdisciplinary Technologies NCU. Author is part of InteRDoCTor project (www.interdoctor.umk.pl).

\section{REFERENCES}

1. Feigin V.L., Norrving B., George M.G., Foltz J.L., Roth G.A., Mensah G.A. Prevention of stroke: a strategic global imperative. Nat Rev Neurol. 201;. doi: 10.1038/nrneurol.2016.107.

2. Kuklina E.V., Tong X., George M.G., Bansil P. Epidemiology and prevention of stroke: a worldwide perspective. Expert Rev Neurother. 2012;12(2):199-208.

3. Strong K., Mathers C., Bonita R. Preventing stroke: saving lives around the world. Lancet Neurol. 2007;6(2):182187.

4. World Health Organization. The Global Burden of Disease: 2004 Update. Geneva, Switzerland: WHO; 2008.

5. Grysiewicz R.A., Thomas Thomas., Pandey D.K. Epidemiology of ischemic and hemorrhagic stroke: incidence, prevalence, mortality, and risk factors. Neurol Clin. 2008;26(4):871-95.

6. Ingall T. Stroke - incidence, mortality, morbidity and risk. J Insur Med. 2004;36(2):143-52.

7. González-Gómez F.J., Pérez-Torre P., DeFelipe A., et al. Stroke in young adults: Incidence rate, risk factors, treatment and prognosis. Rev Clin Esp. 2016; doi: 10.1016/j.rce.2016.05.008.

8. Black-Schaffer R.M., Kirsteins A.E., Harvey R.L. Stroke rehabilitation. 2. Co-morbidities and complications. Arch Phys Med Rehabil. 1999;80(5 Suppl 1):S8-16.

9. Li F, Wu Y., Li X. Test-retest reliability and inter-rater reliability of the Modified Tardieu Scale and the Modified Ashworth Scale in hemiplegic patients with stroke. Eur J Phys Rehabil Med. 2014;50(1):9-15.

10. Gregson J.M., Leathley M., Moore A.P., Sharma A.K., Smith T.L., Watkins C.L. Reliability of the Tone Assessment Scale and the modified Ashworth scale as clinical tools for assessing poststroke spasticity. Arch Phys Med Rehabil. 1999;80(9):1013-6.

11. Hantson L., De Weerdt W., De Keyser J., et al. The European Stroke Scale. Stroke. 1994;25(11):2215-9.

12. Bergmann J., Krewer C., Rieß K., Müller F., Koenig E., Jahn K. Inconsistent classification of pusher behaviour in stroke patients: a direct comparison of the Scale for Contraversive Pushing and the Burke Lateropulsion Scale. Clin Rehabil. 2014;28(7):696-703.

13. Baccini M., Paci M., Nannetti L., Biricolti C., Rinaldi L.A. Scale for contraversive pushing: cutoff scores for diagnosing "pusher behavior" and construct validity. Phys Ther. 2008;88(8):947-55.

14. Karnath H.O., Brötz D. Instructions for the Clinical Scale for Contraversive Pushing (SCP). Neurorehabil Neural Repair. 2007;21(4):370-1; author reply 371.

15. Baccini M., Paci M., Rinaldi L.A. The scale for contraversive pushing: A reliability and validity study. Neurorehabil Neural Repair. 2006;20(4):468-72.

16. Gurcay E., Ozturk E.A., Erdem T., Gurcay A.G., Cakci A. Heterotopic ossification as rare complication of hemiplegia following stroke: two cases. Brain Inj. 2013;27(13-14):1727-31. 
17. Lin S.H., Chiu C.C., Wang C.Y., Chen C.H., Chang K.H. Subdural hygroma-related heterotopic ossification in a patient with a stroke: a case report. Brain Inj. 2011;25(6):624-8.

18. Wissel J., Manack A., Brainin M. Toward an epidemiology of poststroke spasticity. Neurology. 2013;80(3 Suppl 2):S13-9.

19. Siekierka-Kleiser E.M., Kleiser R., Wohlschläger A.M., Freund H.J., Seitz R.J. Quantitative assessment of recovery from motor hemineglect in acute stroke patients. Cerebrovasc Dis. 2006;21(5-6):307-14.

20. Abe H., Kondo T., Oouchida Y., Suzukamo Y., Fujiwara S., Izumi S. Prevalence and length of recovery of pusher syndrome based on cerebral hemispheric lesion side in patients with acute stroke. Stroke. 2012;43(6):1654-6.

21. Karnath H.O., Johannsen L., Broetz D., Küker W. Posterior thalamic hemorrhage induces "pusher syndrome". Neurology. 2005;64(6):1014-9.

22. Pedersen P.M., Wandel A., Jørgensen H.S., Nakayama H., Raaschou H.O., Olsen T.S. Ipsilateral pushing in stroke: incidence, relation to neuropsychological symptoms, and impact on rehabilitation. The Copenhagen Stroke Study. Arch Phys Med Rehabil. 1996;77(1):25-8.

23. Allison R., Shenton L., Bamforth K., Kilbride C., Richards D. Incidence, time course and predictors of impairments relating to caring for the profoundly affected arm after stroke: a systematic review. Physiother Res Int. 2015; doi: 10.1002/pri.1634.

\author{
Address for correspondence: \\ dr Emilia Mikołajewska \\ Department of Physiotherapy \\ Ludwik Rydygier Collegium Medicum in Bydgoszcz \\ Nicolaus Copernicus University \\ ul. M. Curie Skłodowskiej 9 \\ 85-094 Bydgoszcz \\ Poland \\ e-mail: e.mikolajewska@wp.pl,emiliam@cm.umk.pl
}

Received: 20.07.2016

Accepted for publication: 12.08.2016 